\title{
FLORESTAS URBANAS: MÉTODO DE AVALIAÇÃO PARA GESTÃO DAS ÁREAS VERDES
}

\author{
Angelo de Sá Mazzarotto ${ }^{1}$, Selma Cubas $^{2}$, Leila Teresinha Maranho ${ }^{3}$ \\ ${ }^{1}$ Eng. Agrônomo, M.Sc., Universidade Positivo, Curitiba, PR, Brasil - sa@avalon.sul.com.br \\ ${ }^{2}$ Eng ${ }^{\underline{a}}$ Civil, Dr ${ }^{\mathrm{a}}$., Universidade Positivo, Curitiba, PR, Brasil - scubas@up.edu.br \\ ${ }^{3}$ Bióloga, Dra ${ }^{\text {. }}$, Universidade Positivo, Curitiba, PR, Brasil - maranho@up.edu.br
}

Recebido para publicação: 05/11/2008 - Aceito para publicação: 05/05/2011

\begin{abstract}
Resumo
O método proposto neste trabalho tem como objetivo avaliar, pela interação e ponderação, um grupo de indicadores que consideram aspectos quali-quantitativos das florestas urbanas, considerando a arborização em ruas, as praças e os bosques. Para esta proposta, foram levantados indicadores segundo critérios de representatividade na contribuição das florestas urbanas à sociedade quanto aos aspectos estéticos, qualidade do ar, controle de enchentes e temperatura. Estes foram atestados em sua confiabilidade e representatividade e agrupados em dois conjuntos: macro e microambientais. $\mathrm{O}$ primeiro é composto por aspectos capazes de avaliar características mais amplas, inerentes a um conjunto de indivíduos, e o segundo, por características do próprio indivíduo vegetal, como aspecto da raiz. Aos indicadores foram atribuídos pesos, diferenciando-os em suas representatividades dentro do método. Como resultado, obteve-se um método que utiliza o barômetro da sustentabilidade para a interseção de suas respostas em dois eixos (macro e microambientais), fornecendo um diagnóstico da sua condição de sustentabilidade. Como exemplo de aplicação, a proposta foi testada nas áreas do campus da Universidade Positivo e do Jardim Botânico, ambas localizadas na cidade de Curitiba, Paraná, e mostrou-se adequada para diagnosticar as condições de sustentabilidade.
\end{abstract}

Palavras chave: Arborização; indicadores ambientais; barômetro da sustentabilidade.

\begin{abstract}
Urban forests: evaluation method to management of green area. This method aims to evaluate a group of indices by interaction and balance; it considers qualitative as well as quantitative aspects of Urban Forests for evaluation of elements such as: forests, wooden streets and wooden squares. In order to develop such proposal, several indices had been selected from specialized literature, mainly the ones that reveal the contribution of Urban Forests to society in relation to aesthetic aspects, quality of air, as well as flood and temperature control. Such indices had been certified in relation to their trustworthiness and relevance and they had been grouped in two sets: macro and micro ambient. The first group was composed to evaluate wider characteristics that are inherent to a set of individuals; the second one was proposed to evaluate characteristics of the vegetal individual itself, such as aspects of the root. Different weight had been attributed to the indices, in order to differentiate them by their relevance up to the method. As result it produced a method that uses a sustainability barometer for intersection of two axes, macro and micro ambient, in order to diagnose sustainability condition. It had been tested in two different areas, campus of Positivo University and Botanical Garden, both located in Curitiba, Paraná, and it proves as able to the sustainability condition. Keywords: Arborization; environment pointers; sustainability barometer.
\end{abstract}

\section{INTRODUÇÃO}

O crescimento desordenado das cidades está causando uma grande redução da cobertura arbórea, afetando a qualidade de vida da população. A escassez de áreas verdes associada à instabilidade climática e à poluição (atmosférica, hídrica, sonora e visual) afeta a qualidade de vida das pessoas, provocando distúrbios biológicos e psicológicos (CARVALHO, 2001).

Para a gestão dos problemas ambientais nos núcleos urbanos, é imprescindível o manejo da vegetação, que, por sua vez, se constitui num grande desafio do planejamento urbano. Conforme Badiru 
et al. (2005), a inexistência de um manejo planejado ecologicamente e integrado ao ambiente urbano faz com que as florestas urbanas se apresentem, em muitas situações, mal estruturadas e desordenadas, o que promove a sua deterioração. Milano e Dalcin (2000) afirmam que a falta de planejamento é um dos fatores mais limitantes para a manutenção e o desenvolvimento dessas florestas.

Segundo Jesus e Braga (2005), é essencial para a recuperação e potencialização de aspectos positivos que sejam consideradas as interações entre as formas urbanas e a natureza, e que elas sejam as mais harmoniosas possíveis. Sendo assim, é imprescindível que o planejamento e a gestão de florestas urbanas levem em consideração as relações entre os aspectos e indicadores sociais e ambientais como conceitos associados à quantidade, qualidade e distribuição, que, na maioria das vezes, são analisados isoladamente.

Um estudo realizado por Silva (2000) demonstrou que significativos problemas ambientais quanto à arborização urbana decorrem da falta de planejamento e que tal situação é identificada em várias cidades brasileiras, conforme seu levantamento. No Brasil há muitas cidades arborizadas, no entanto grande parte não possui um planejamento e, em consequência, apresenta sérios problemas de manejo e gestão dessas áreas.

A necessidade do manejo das vegetações nos núcleos urbanos tem sido um dos mais agudos desafios, tendo em vista o acúmulo de problemas ambientais nos últimos tempos, afetando os recursos naturais $\mathrm{e}$ os equipamentos urbanos, influenciados negativamente, principalmente nas zonas metropolitanas e regiões conurbadas (MILANO, 1988).

O ponto mais importante no processo de planejamento global da arborização urbana de uma cidade é enxergá-la no contexto holístico (PAIVA; GONÇALVES, 2002). É imprescindível, portanto, adotar estratégias ambientalmente seguras, especialmente voltadas às cidades, que favoreçam a sua gestão. Nesse sentido, é necessário analisar o padrão e a forma urbana e atender as premissas estabelecidas para um desenvolvimento sustentável, com o intuito de favorecer a manutenção da qualidade da vida da população.

De forma consensual entre os pesquisadores de florestas urbanas, seus benefícios são indiscutíveis. As árvores interceptam, absorvem, refletem, captando e transpirando água. Interferem também na velocidade e direção dos ventos, atuando de forma efetiva na melhoria do microclima urbano e promovendo uma melhoria na qualidade de vida urbana, assim como no conforto visual da população pela sua influência paisagística (TAKAHASHI, 1994).

Convergir às diversas variáveis representadas pelos indicadores, usualmente utilizadas na literatura para diagnosticar os parâmetros quali-quantitativos em uma única referência, não apenas facilitará o diagnóstico por gestores como também possibilitará a compreensão da interação dos aspectos relacionados às florestas urbanas e sua importância.

Na busca da real avaliação diagnóstica das áreas verdes urbanas, vários aspectos são levados em consideração, separados ou em conjuntos. Oliveira (1996) comenta que alguns trabalhos fazem levantamento e cadastramento de árvores com o objetivo de avaliar as condições fitossanitárias da vegetação, enquanto outros buscam avaliar a utilização do espaço pelo público e os aspectos percebidos pela população em relação à arborização.

O presente trabalho teve como objetivo desenvolver um método de avaliação das florestas urbanas baseado na busca e seleção de indicadores em literatura especializada, assim como na relação quali-quantitativa entre eles. Esse método foi, ainda, testado em duas áreas verdes na cidade de Curitiba, PR, Brasil.

\section{MATERIAL E MÉTODOS}

Foram empregados no presente estudos dois termos principais - grupos temáticos e indicadores ambos adotados a partir do conceito descrito por Tomasoni (2006).

\section{Proposta dos grupos temáticos}

Nos grupos temáticos, assumiu-se a situação de abrangência como a impossibilidade de tal condição ser avaliada por um único critério ou parâmetro. Já os indicadores foram considerados uma importante ferramenta de análise espacial. Devem ser tomadas, entretanto, precauções quanto ao emprego das terminologias associadas, como indicador, subíndice e índice, respeitando a sua hierarquia. 
Esses indicadores foram propostos por serem representativos quanto ao potencial de contribuição socioambiental das florestas urbanas, o qual está relacionado às características do indivíduo arbóreo, bem como do conjunto, representado na mesma ordem por aspectos micro e macroambientais. Os aspectos macroambientais agrupam características observadas no conjunto de indivíduos, e os aspectos microambientais agrupam características observáveis somente no indivíduo.

Os indicadores propostos foram incluídos em grupos temáticos por serem expressos em forma de temas, os quais representam um ou mais potenciais contributivos, sendo elaborados nesta etapa um ou mais temas para cada uma das condições apresentadas: de estado, de pressão e de resposta.

\section{Seleção dos indicadores}

A seleção dos indicadores baseou-se na afinidade das características observáveis das florestas urbanas expressas em seus grupos temáticos.

Após seleção, esses indicadores foram agrupados aos grupos temáticos segundo os parâmetros que compõem cada um. Esses parâmetros representaram os temas dos indicadores.

Validação dos indicadores

Após análise de relevância, com bases em artigos científicos, teses e dissertações, solicitou-se por e-mail, a 220 profissionais de todo o Brasil com afinidade na área deste estudo (pesquisadores, professores e especialistas, cujos endereços eletrônicos foram obtidos em sites de universidades e da Sociedade Brasileira de Arborização Urbana), o preenchimento de uma tabela para avaliação de indicadores. Esses profissionais foram informados quanto aos objetivos da pesquisa e forma de preenchimento: ao lado de cada indicador, deveria ser atribuída uma nota de 1 a 10. Além disso, solicitouse também a inclusão de indicadores que, porventura, eles considerassem importantes e que não foram contemplados na seleção.

Valoração dos indicadores

Os indicadores foram submetidos a um processo de valoração por meio da atribuição de notas de 1 a 10. Os valores de cada nota determinaram a representatividade de cada indicador e serviram como critério para a composição do método de avaliação. A atribuição das notas baseou-se em dois critérios propostos por Braga et al. (2004a) e nas notas atribuídas pelos profissionais que responderam a pesquisa, totalizando 9 dos 220 solicitados para o preenchimento da tabela de indicadores.

As notas atribuídas correspondem à influência dos indicadores nas seguintes características segundo proposta de Braga et al. (2004a):

- Relevância - potencialidade do índice em representar ou traduzir o fenômeno.

- Aderência local - potencialidade do índice em captar fenômenos produzidos no local.

- Disponibilidade - abrangência e atualidade dos dados.

- Historicidade - potencialidade dos dados em permitir comparações temporais.

A nota de cada uma dessas características variou de 2-2,5, podendo atingir uma nota total de 10 para cada indicador caso todas as características tenham sido consideradas com nota 2,5. As demais possibilidades dependeram de quantas características foram atribuídas e de suas notas. Na não representatividade dos indicadores por essas características, ele será desconsiderado.

Análise dos dados recebidos pelos profissionais

Os dados coletados foram submetidos a uma média aritmética para obtenção de uma única nota para cada índice. Dessa forma, todos os valores foram somados e divididos pelo total de vezes em que esse índice foi avaliado. Os desvios não foram considerados, pois o número de respostas foi inferior a $10 \%$ do total de respostas esperadas, tomando por base o trabalho realizado por Lima et al. (1994), quando foram obtidas 20 das 192 respostas esperadas.

Após aplicação da média aritmética, os indicadores obtiveram valores entre 0 e 10 , que expressam o grau de representatividade de cada índice para com o seu grupo temático. 
Obtenção dos indicadores

A seleção final dos indicadores que compõem o método de avaliação foi obtida pela média ponderada dos valores atribuídos a cada um. Nessa média, os valores atribuídos pelos profissionais tiveram um peso igual a 6 , para lhes proporcionar uma maior influência na composição do método em relação aos atribuídos conforme critérios de Braga et al. (2004a), que tiveram peso 4. Após a média ponderada, os indicadores que obtiveram nota inferior a 6 foram eliminados. Esse valor foi adotado como nota mínima de seleção, o que representa $60 \%$ da nota máxima possível.

\section{Padronização}

Uma escala numérica composta dos valores $0,1,3,5,7,9$ e 10 foi adotada como procedimento para padronizar os resultados expressos para cada um dos indicadores que compõem o método. A padronização foi feita porque os indicadores apresentam unidades e proporções diferentes, impossibilitando uma única leitura.

Na padronização também foram estabelecidos valores máximos e mínimos, que representam as condições desejadas e indesejadas na qualidade das florestas urbanas. Esses valores são representados pelos algarismos 1 e 9 . Os números 0 e 10 representaram as condições que tendem ao ideal ou a pior possível, como, por exemplo, situação de floresta densa ou desertificação total. Os algarismos 3, 5 e 7 representaram as diversas possibilidades de resultados entre as condições determinadas pelos valores máximos e mínimos.

\section{Valores máximos e mínimos}

Os valores máximos e mínimos foram adotados para expressar duas condições - desejada e indesejada, representadas pelos algarismos 1 e 9. Esses valores representaram condições apontadas na literatura como ruins ou desejáveis, tendo sempre uma relação com valores apresentados pelos indicadores.

O procedimento adotado abrange duas situações possíveis. Na primeira, considera-se um dos valores máximo ou mínimo encontrados na literatura relacionados aos termos: condição desejável, ruim ou análoga; o outro valor será determinado, considerando sempre o valor mínimo $50 \%$ menor. Na segunda situação, nenhum valor relacionado aos limites máximos e mínimos foi encontrado, então foi proposto um dos valores com base na interpretação e dados levantados em diagnósticos apresentados em artigos científicos, respeitando-se a condição anterior.

\section{Leitura dos resultados utilizando o barômetro da sustentabilidade}

A utilização do barômetro da sustentabilidade finaliza o método de avaliação. Para a incorporação dessa ferramenta, foi necessária a modificação da estrutura apresenta por Braga et al. (2004a). Essas alterações consistiram em:

- Modificações dos eixos, para que representassem os dois conjuntos de indicadores propostos, que englobam as condições macro e microambientais.

- Adaptação das escalas dos eixos. Essa alteração mantém o valor 0 como inicial, determinando como valor máximo o maior resultado possível atingido pelo conjunto de indicadores em seus respectivos eixos. $\mathrm{O}$ intervalo entre esses dois valores foi dividido em quatro partes, possibilitando 16 posições e quatro diagnósticos: Sustentável; Potencialmente sustentável; Potencialmente insustentável; Insustentável. Essa divisão se apoia no conceito de quartil, também conhecido por separatrizes. A divisão da escala proposta no barômetro da sustentabilidade em quatro partes, pelos três quartis $\mathrm{Q}_{1}$, $\mathrm{Q}_{2}$ e $\mathrm{Q}_{3}$, dividiu o conjunto dos dados em quatro subconjuntos, de tal forma que $25 \%$ dos elementos que representam o primeiro diagnóstico situam-se abaixo do $\mathrm{Q}_{1} ; 25 \%$ entre $\mathrm{Q}_{1}$ e $\mathrm{Q}_{2} ; 25 \%$ entre $\mathrm{Q}_{2} \mathrm{e}$ $\mathrm{Q}_{3} ;$ e $25 \%$ acima de $\mathrm{Q}_{3}$, sendo que $\mathrm{Q}_{2}$ corresponde à mediana.

\section{Aplicação do método de avaliação nas áreas-teste}

Para testar o método, foram selecionadas duas áreas na cidade de Curitiba-PR: o Jardim Botânico e o Campus da Universidade Positivo. As áreas foram escolhidas por apresentarem diversos elementos componentes das florestas urbanas, como arborização de ruas, bosques, vegetação de pequeno e médio porte e áreas edificadas. 
A coleta de dados

A coleta de dados foi feita através de ortofotos, inventários com a utilização de fichas para coleta de dados com adaptações (MELO, 2007), questionários aplicados aos frequentadores das duas áreas e levantamento fitossociológico da vegetação fanerógama do Jardim Botânico, realizado por Nascimento (2005).

As ortofotos, fotos aéreas digitalizadas, possibilitaram a obtenção de dados como dimensões, perímetros e distâncias. As ortofotos foram fornecidas pelo Instituto de Pesquisa e Planejamento Urbano de Curitiba (IPPUC). Para o tratamento das ortofotos, foi utilizado o software ArcView GIS 3.2a.

Para o inventário quali-quantitativo das áreas, foram realizadas amostragens aleatórias. A técnica utilizada consistiu em estimar os dados com auxílio de trena, fita métrica e observações a olho nu. Essa técnica, segundo Silva et al. (2006), é de rápida aplicação e com utilização de menos recursos.

A coleta de dados pelo método de inventário complementou as informações necessárias sobre a qualidade microambiental, como, por exemplo, condição de sanidades, raízes e outras requeridas nos indicadores empregados. Para essa etapa, foi utilizada uma ficha de coleta de informação de campo, adaptada do modelo para diagnóstico utilizado por Melo et al. (2007).

As informações foram coletadas aleatoriamente, buscando-se atingir a totalidade das áreas analisadas. Foram analisados 85 indivíduos em cada uma das áreas de testes.

O questionário foi aplicado para coleta de informações referentes ao valor de contingência. Os entrevistados foram questionados nas seguintes informações: nome, idade, localidade, escolaridade e sua disponibilidade de pagar qualquer quantia acima de $\mathrm{R} \$ 1,00$. $\mathrm{O}$ valor proposto tem por base a média de R \$ 1,21 obtida por Hildebrand (2001) na avaliação econômica de áreas verdes.

Com referência no total de entrevistados por Hildebrand (2001) no "Bosque do Alemão", em Curitiba, foram aplicados ao todo 30 questionários, 15 em cada uma das áreas.

Para diagnosticar a estrutura da vegetação fanerógama da área do Jardim Botânico, foram utilizados dados fitossociológicos, como densidade, dominância, frequência e valor de importância, obtidos por Nascimento (2005).

\section{RESULTADOS E DISCUSSÃO}

\section{Proposta dos grupos temáticos}

Os temas para os indicadores foram propostos com o objetivo de abranger as características que são avaliadas nas florestas urbanas. Como resultado dessa etapa, foram constituídos quatro grupos temáticos:

- Qualidade dos componentes das florestas urbanas;

- Qualidade dos indivíduos;

- Pressão antrópica na composição das florestas urbanas;

- Contribuição econômica das florestas urbanas.

Qualidade dos componentes das florestas urbanas: agrupou indicadores de estado que medem aspectos relacionados à qualidade das florestas urbanas. As variáveis ordenadas por esses indicadores analisaram a presença dos componentes dessas áreas, como parques, praças e maciços arbóreos. Essas variáveis refletem o alcance das funcionalidades associada à proteção contra erosão, à redução de enchentes, à promoção do lazer e à integração social.

Qualidade dos indivíduos: agrupou indicadores de estado que medem aspectos relacionados à qualidade dos indivíduos pertencentes às florestas urbanas. As variáveis ordenadas por esses indicadores medem as condições individuais, como ocorrência de depredação e necessidade de podas e doenças.

Pressão antrópica na composição das florestas urbanas: agrupou indicadores de pressão que medem o estresse exercido sobre as florestas urbanas locais pela interação antrópica, referentes aos processos de urbanização e às atividades econômicas. Essas influências podem ser negativas e positivas e estão associadas ao número de pessoas, às edificações e às áreas de impermeabilização.

Contribuição econômica das florestas urbanas: agrupou indicadores de resposta que medem a capacidade das florestas urbanas em prestar serviços aos sistemas político, institucional, social, cultural e a própria percepção da sociedade sobre a contribuição paisagística, o controle de enchentes, a poluição e o lazer. 


\section{Seleção de indicadores}

Os indicadores selecionados foram levantados em vários artigos científicos e utilizados como referências para a composição da tabela 1 . Esses indicadores foram encontrados na literatura relacionados às florestas urbanas ou termos correlatos.

Tabela 1. Indicadores identificados segundo critério de relação a florestas urbanas e seus elementos.

Table 1. Identified indicators as criterias of relation between urban forests and its elements.

\begin{tabular}{lcc}
\hline Indicadores & Composição dos indicadores & Fonte \\
\hline IAUrb & Total de áreas verdes com número populacional & Berté (2006) \\
Di & Porcentagem da espécie mais frequente & Milano (1988) \\
IAVU & Total de áreas verdes utilizáveis & Fialho et al. (2006) \\
PAV & Relação entre arborização e cobertura vegetal rasteira & Silva (2004) \\
ICV & Relação entre área de copa por habitante & Braga et al. (2004a) \\
ELH & Avalia espaços livres herbáceos & Silva (2005) \\
IFU & Relação entre arborização e espaço intraurbano & Moreira et al. (2007) \\
PAI & Proporção entre espaços arborizados e espaços livre impermeável & Moreira et al. $(2007)$ \\
PAC & Proporção entre espaços arborizados e espaços construídos & Moreira et al. $(2007)$ \\
DP & Densidade populacional & Berté (2006) \\
VC & Valor contingente & Hildebrand (2001) \\
CI & Contribuição monetária de cada indivíduo & Hildebrand (2001) \\
VImp & Valor de implantação & Detzel (1992) \\
Necessidade de podas & Qual a necessidade de poda & Melo et al. (2007) \\
Desenvolvimento & Altura da primeira bifurcação, DAP & Melo et al. $(2007)$ \\
Espaçamento & Distância entre árvores & Milano (1988) \\
Sanidade & Presença de injúrias ou doenças & Milano (1988) \\
Adubação & Quantas fases de adubação & Seitz (1995) \\
Condição da raiz & Sadia, exposta ou danificada & Melo et al. $(2007)$ \\
Condição da copa & Normal, avançando & Melo et al. $(2007)$ \\
\hline
\end{tabular}

Relação entre grupos temáticos e indicadores

No grupo temático "Qualidade das Florestas Urbanas", os indicadores avaliaram aspectos relacionados à arborização sem considerar o ser humano. Os indicadores são: percentual de áreas verdes (PAV), diversidade (Di), espaços livres herbáceos (ELH), estratificação e valor de importância das espécies (VI). O diagnóstico através da utilização desses parâmetros possibilitou a identificação das condições macroambientais.

No grupo temático "Pressão Antrópica", os indicadores adotados foram: indicadores de áreas verdes urbanas (IAVUrb), índice de cobertura vegetal (ICV), índice de florestas urbanas (IFU), densidade populacional (DP), proporção entre espaços arborizados e impermeabilizados (PAI), índice de áreas verdes utilizáveis (IAVU), proporção entre espaços arbóreos com espaços construídos (PAC). Tais indicadores buscaram verificar a influência da pressão antrópica nas florestas urbanas, através da relação entre as áreas verdes e o espaço intraurbano e população. Ou seja, diagnosticam o quanto a população interfere negativamente na sustentabilidade das florestas urbanas e utilizam dados relacionados a grupos de indivíduos vegetais. Por essa razão, são classificados como macroambiental.

No grupo temático "Contribuição Econômica das Florestas Urbanas", os indicadores medem a capacidade das florestas urbanas de prestar um serviço à sociedade, sendo mensurável e/ou percebido pela população. A esse tema foram relacionados indicadores que expressam a disposição da população em pagar por um serviço percebido, a economia gerada frente a controles de enchentes, poluição, custo de implantação das áreas verdes e valor de um indivíduo vegetal, estimado pelo seu porte, idade e outros valores intrínsecos. Os índices relacionados são: valor de contingência (VC), contribuição do indivíduo (CI) e valor de implantação (VImp).

No grupo temático "Qualidade do Indivíduo", os indicadores apontam a qualidade das florestas urbanas com o foco no indivíduo, independentemente da abrangência dos mesmos, permitindo uma interpretação precisa do estado quali-quantitativo, não valorizando somente a presença dos elementos, 
mas o estado em que se encontram. Nesse tema foram observadas condições como necessidade de poda, sanidade, adubação, condição da raiz e condição da copa.

Validação dos indicadores para a composição do modelo

Os indicadores da tabela 1 foram analisados segundo os critérios estabelecidos por Braga et al. (2004a), que são relevância (R), aderência local (A), disponibilidade (D) e historicidade (H). Para essa etapa, foram atribuídos pontos aos indicadores, considerando a presença das características, com notas variando de 2-2,5 para cada uma das características atribuídas, conforme tabela 2, na coluna "Pontuação atribuída segundo R A D H".

Avaliação das tabelas respondidas pelos professores, pesquisadores e especialistas

Dos 220 questionários enviados, apenas 9 foram respondidos com os indicadores avaliados. Devido ao baixo número de respostas, as notas foram submetidas a média aritmética sem cálculo de desvios. Na tabela 2, na coluna "Notas atribuídas pela pesquisa", são apresentadas as médias das notas recebidas.

Ponderação dos indicadores

Nessa etapa, foi adotado o seguinte critério: os valores apresentados na coluna da "Pontuação atribuída segundo R A D H" foram multiplicados pelo peso 4, e os da coluna "Notas atribuídas pela pesquisa" por 6 , seguidos de uma soma, índice por índice, e divididos por 10. Dessa forma, foi atribuída uma maior influência às avaliações realizadas pelos profissionais. Na última coluna está apresentada a pontuação obtida pela média, sendo considerados para a próxima etapa os índices com nota superior a 6 .

Tabela 2. Composição das notas dos indicadores e pontuação atingida pela média ponderada.

Table 2. Composition of notes of the indicators and punctuation reached for the weighed average.

\begin{tabular}{lcccc}
\hline Indicadores & $\begin{array}{c}\text { Notas atribuídas } \\
\text { pela pesquisa }\end{array}$ & \multicolumn{2}{c}{$\begin{array}{c}\text { Pontuação atribuída } \\
\text { segundo R A D H }\end{array}$} & $\begin{array}{c}\text { Pontuação obtida através } \\
\text { da média ponderada }\end{array}$ \\
\hline IAUrb & 6,3 & 10,0 & R A D H & $7,8^{*}$ \\
Diversidade & 6,3 & 10,0 & R A D H & $7,8^{*}$ \\
PAV & 4,6 & 8,5 & RAD H & 6,2 \\
ICV & 3,0 & 2,0 & H & 2,6 \\
ELH & 5,8 & 4,0 & AH & 5.1 \\
IFU & 7,5 & 9,5 & R A D H & $8,3^{*}$ \\
DP & 6,5 & 9,5 & R A D H & $7,7^{*}$ \\
PAI & 8,0 & 2,5 & D & 5,8 \\
IAVU & 5,3 & 6,0 & R A D & 5,6 \\
PAC & 7,0 & 4,0 & D H & 5,8 \\
VC & 6,0 & 8,0 & R A D H & $6,8^{*}$ \\
CI & 0,0 & 0,0 & - & - \\
VImp & 8,5 & 2,0 & H & 5,9 \\
Necessidade de podas & 8,0 & 8,0 & R A D H & $8,0^{*}$ \\
Desenvolvimento & 7,0 & 10,0 & R A D H & $8,2^{*}$ \\
Espaçamento & 4,0 & 6,0 & R D H & 4,8 \\
Sanidade & 8,0 & 10,0 & R A D H & $8,8^{*}$ \\
Adubação & 7,0 & 4,0 & R D & 5,8 \\
Condição da raiz & 8,0 & 5,0 & R A & 6,8 \\
Condição da copa & 8,0 & 2,0 & D & 5,6 \\
\hline
\end{tabular}

Pontuação variando de 1 a 10. *: Indicadores que obtiveram nota superior e igual a 6 nos dois processos de valoração. D: disponibilidade; R: Relevância; H: Historicidade; A: aderência.

\section{Obtenção do método}

De um total de 23 indicadores que compõem as tabelas de seleção, 10 foram selecionados para compor o método, pois obtiveram notas igual ou maior à nota mínima estabelecida de 6 pontos nessa fase. Cabe ressaltar que 8 dos 10 indicadores selecionados obtiveram nota acima da linha de corte nos dois métodos de avaliação. 
Ainda para a composição do método, foi feita a transferência da representatividade atribuída aos indicadores pelas notas. Dependendo do valor da nota atribuída aos indicadores, foram estabelecidos pesos que variaram entre 1 e 4: para valores obtidos de 6 a 7 peso (1), de 7,1 a 8 peso (2), de 8,1 a 9 peso (3), de 9,1 a 10 peso (4), ampliando-se, dessa forma, a representatividade dos indicadores selecionados.

Na tabela 3 estão apresentados os indicadores selecionados para compor o método, as notas obtidas e os pesos atribuídos, divididos em dois grupos, que correspondem aos eixos macro e microambiental.

Tabela 3. Notas e pesos dos indicadores que compõem o modelo de avaliação.

Table 3. Notes and weights of the indicators that encompasses the model of evaluation.

\begin{tabular}{lccc}
\hline Indicadores & Pontuação 1 a 10 & Pesos & Aspectos ambientais \\
\hline IAUrb & 7,8 & 2 & Macro \\
Diversidade & 7,8 & 2 & \\
PAV & 6,2 & 1 & \\
DP & 7,7 & 2 & \\
IFU & 8,3 & 3 & Micro \\
VC & 6,8 & 1 & \\
Necessidade de podas & 8,0 & 2 & \\
Desenvolvimento & 8,2 & 3 & \\
Sanidade & 8,8 & 3 & \\
Condição da raiz & 6,8 & 1 & \\
\hline
\end{tabular}

Na etapa final, o método resultou em duas equações (1 e 2), cujos resultados serão utilizados no barômetro de sustentabilidade para avaliação da sustentabilidade das florestas urbanas.

A primeira equação (1) foi composta por indicadores dos grupos temáticos Qualidade das Florestas Urbanas e Pressão Antrópica na Composição das Florestas Urbanas. Na segunda equação (2), os indicadores ficaram compreendidos nos grupos temáticos Qualidade dos Indivíduos e Contribuição Econômica das Florestas Urbanas.

$$
\begin{aligned}
& \mathrm{VMaA}=3 \mathrm{IFU}+2 \mathrm{IAUrb}+2 \mathrm{Di}+2 \mathrm{DP}+\mathrm{PAV} \\
& \mathrm{VMiA}=3 \mathrm{De}+3 \mathrm{Sa}+2 \mathrm{~Np}+\mathrm{Cr}+\mathrm{VC}
\end{aligned}
$$

Os indicadores que compõem o método foram descritos segundo suas características qualiquantitativas, seu estado de pressão ou estado ou respostas e seus parâmetros avaliáveis dentro das florestas urbanas.

Índice de áreas verdes urbanas (IAUrb)

Propõe uma relação direta da quantidade de áreas verdes urbanas com a população total do local analisado. É indicativo quantitativo e de pressão, por estar relacionado com a presença dessas áreas e a influência exercida pelo tamanho da população residente. Foi selecionado por possibilitar um dado macroambiental de interpretação direta, de possível obtenção, por permitir uma avaliação temporal, uma aplicabilidade em qualquer região e as possíveis comparações entre outras áreas.

Para a sua utilização, é necessária a quantificação total das áreas verdes urbanas e o total da população residente, desconsiderando a influência da população flutuante, conforme Berté (2006).

O dado obtido por esse indicador é expresso $\mathrm{em}^{2}$ por habitante, tendo como um bom índice a relação de $15 \mathrm{~m}^{2}$.hab ${ }^{-1}$, conforme referência apresentado pela ONU e SBAU, também utilizado por Berté (2006).

\section{Diversidade (Di)}

Esse índice expressa a diversidade de espécies em uma determinada área, demonstrando a frequência das espécies e a relação entre elas, um dado importante para o planejamento de florestas urbanas, conforme Milano (1988). Lima et al. (2007) atribuem à homogeneidade acentuada de indivíduos nas áreas verdes o risco de perda por eventuais ataques de pragas.

Para esse índice, foi adotada a recomendação de Grey e Deneke (1986), que afirmam que frequências maiores que $15 \%$ de uma espécie passam a apresentar uma intensificação nos problemas fitossanitários e perda na estética, segundo critérios paisagísticos. 
Relação entre arborização e cobertura vegetal rasteira (PAV)

Esse índice expressa a porcentagem da vegetação arbórea e da vegetação rasteira presentes nas áreas verdes urbanas.

$\mathrm{Na}$ escolha dos valores extremos para esse índice, levou-se em consideração a potencialidade das florestas urbanas sob critérios paisagísticos, ecológicos, de barreiras sonoras e de valoração mobiliária, que tendem a aumentar com o porte da vegetação das florestas urbanas. Por essa razão, quanto mais as áreas verdes urbanas forem compostas por espécies arbóreas de maior porte, maior será o seu potencial contributivo.

Foi adotada como valor mínimo para esse índice uma relação entre vegetação arbórea e vegetação rasteira igual a 1/3. Essa relação foi obtida pela interpretação da avaliação realizada por Ferreira e Gontijo (2005).

Indicadores de florestas urbanas (IFU)

O índice expressa a proporção entre espaço arborizado, com espaço livre impermeável, e espaço construído. Essa relação demonstra a influência de espaços cobertos por calçadas, passarelas, ruas e construções. Sua importância está na possibilidade de analisar áreas impermeáveis por construção ou áreas impermeáveis por pavimentação. IFU sofre influência direta de legislação municipal, que determina áreas mínimas permeáveis em edificações.

Para indicação dos valores extremos, foram adotadas as porcentagens de $25 \%$ de área permeável exigidas para edificações pela Prefeitura Municipal de Curitiba, conforme decreto $\mathrm{n}^{\circ} 791$, de 2003, para o coeficiente/taxa de permeabilidade exigido para o município, exceto em lotes inferiores a $360 \mathrm{~m}^{2}$, situados em setor especial de habitação de interesse social (SEHIS).

Densidade populacional (DP)

$\mathrm{O}$ índice densidade populacional influencia profundamente as características e condições de qualidade das florestas urbanas. Por essa razão, esse índice foi considerado fundamental no método de avaliação proposto neste trabalho, pois relaciona o aumento da densidade demográfica com a perda da qualidade ambiental.

Para a proposição dos valores extremos e indicação dos intervalos, foi adotada a recomendação expressa na Carta de Densidade Demográfica elaborada por Souza et al. (2004), que propõem como desejável um valor inferior 400 hab.ha $^{-1}$.

Valor de contingência (VC)

$\mathrm{O} \mathrm{VC}$ foi selecionado por estar relacionado às atribuições das florestas urbanas, tais como a capacidade de interação no lazer da população, a acessibilidade dessas áreas pela comunidade e o reconhecimento do seu valor.

O valor de contingência foi obtido pela identificação da disposição da comunidade em pagar ou estimar um valor financeiro de um determinado elemento que compõe a floresta urbana. Porém, para a sua utilização na composição do método, o valor atribuído não foi usado como um indicativo, pois, segundo Hildebrand (2004), os valores atribuídos têm uma forte relação com a remuneração do entrevistado, como também sua classificação como turista ou morador da comunidade. Dessa forma, o índice aponta o interesse ou desinteresse na disposição a pagar descrevendo, assim, um intervalo entre o sim, o talvez e o não.

O benefício social e ambiental que são percebidos como contribuição ao bem-estar da comunidade pode ser relacionado a um valor ou à disposição em pagar, segundo a teoria microeconômica do bem-estar, conforme Motta (1998).

O uso desse índice não só aponta o grau do reconhecimento das contribuições das florestas urbanas pela comunidade, como também propõe indicativos do acesso da comunidade a essas áreas.

Necessidade de poda $(\mathrm{Np})$

O índice avalia a condição de estado, promovendo um diagnóstico da arquitetura da planta, em função de suas condições fisiológicas e sua interação com a edificação urbana.

Para $\mathrm{Np}$ foi adotado como referência uma condição desejável de $75 \%$ ou mais não necessitando poda. Esse valor foi apresentado por Melo et al. (2007). 
Desenvolvimento (De)

O índice faz uma relação entre a maturidade dos indivíduos componentes das florestas urbanas e a sua capacidade contributiva, considerando que quanto maior o seu estado de maturidade maior a sua eficiência. Portanto, torna-se necessário a consideração do estado de desenvolvimento dessas áreas.

Para estipulação dos valores extremos, foi utilizado como referência o diâmetro de $15 \mathrm{~cm}$, medido na altura do peito (DAP), que é igual a 1,3 m. Como valor desejável, adotou-se uma porcentagem mínima de $45 \%$ dos indivíduos que possuam valores iguais ou superiores ao de referência. Esse valor foi adotado segundo critério indicado pela Prefeitura Municipal de Curitiba.

Sanidade (Sa)

Para esse índice, foi adotado como referência o estudo de Bortoleto (2004), que considerou um bom estado de sanidade valor igual a $75 \%$ sem enfermidade. Esse índice está relacionado à seguinte condição do indivíduo: presença de enfermidades, lesões, pragas e arquitetura não adequada.

Condição da raiz $(\mathrm{Cr})$

Esse índice foi selecionado por apontar, entre outros, dados importantes sobre gestão de áreas verdes e condição fitossanitária do indivíduo, o primeiro relacionado à manutenção de calçadas, e o segundo, desenvolvimento e fixação. Para que essas variáveis se apresentem em condições desejáveis, torna-se significativa a condição apresentada pelas raízes. Para estipulação dos valores extremos, foram adotados os valores apresentados por Melo et al. (2007), que consideraram como desejável $95 \%$ da população analisada com raízes sem injúrias ou afloramentos significativos.

\section{Descrição dos intervalos, proposição dos valores extremos}

Os valores extremos expressam um intervalo entre duas condições de qualidades - desejável ou indesejável, que foram adotadas conforme dados obtidos na literatura, as quais avaliaram as florestas urbanas ou os indivíduos presentes nessas áreas. Como os valores apresentam unidades e escalas diferentes, foi adotada uma escala de padronização para os indicadores com valores iguais a $0,1,3,5,7,9$ e 10. Na tabela 4 estão apresentados os valores extremos e seus intervalos.

Tabela 4. Relação entre indicadores e os valores extremos e escala de parâmetros.

Table 4. Relation between indicators and the extreme values and scale of parameters.

\begin{tabular}{|c|c|c|c|c|c|c|c|}
\hline Pesos & (0) & (1) & (3) & (5) & (7) & (9) & (10) \\
\hline Indicadores & $\begin{array}{c}\text { Fora do } \\
\text { limite de } \\
\text { referência }\end{array}$ & $\begin{array}{c}\text { Valor } \\
\text { mínimo }\end{array}$ & & \multicolumn{2}{|c|}{ Intervalos } & $\begin{array}{l}\text { Valor } \\
\text { máximo }\end{array}$ & $\begin{array}{c}\text { Fora do } \\
\text { limite de } \\
\text { referência }\end{array}$ \\
\hline IAUrb & $\begin{array}{c}<7,5 \mathrm{~m}^{2} / \\
\text { habitante }\end{array}$ & $\begin{array}{c}7,5 \mathrm{~m}^{2} / \\
\text { habitante }\end{array}$ & 9,3 & 11,3 & 13,0 & $\begin{array}{c}15 \mathrm{~m}^{2} / \\
\text { habitante }\end{array}$ & $\begin{array}{c}>15 \mathrm{~m}^{2} / \\
\text { habitante }\end{array}$ \\
\hline Diversidade & $>22,5 \%$ & $22,5 \%$ & 20,7 & 18,8 & 16,9 & $\begin{array}{l}15 \% \text { mais } \\
\text { frequente }\end{array}$ & $\begin{array}{l}<15 \% \text { mais } \\
\text { frequente }\end{array}$ \\
\hline IFU & $<15 \%$ & $15 \%$ & 18,8 & 22,5 & 26,3 & $30 \%$ & $>30 \%$ \\
\hline PAV & $<15 \%$ & $15 \%$ & 18,8 & 22,5 & 26,3 & $30 \%$ & $>30 \%$ \\
\hline DP & $>800$ & 800 & 550 & 500 & 450 & $\begin{array}{c}400 \\
\text { hab.ha }^{-1}\end{array}$ & $<400$ \\
\hline $\mathrm{VC}$ & $<25 \%$ & $25 \%$ & 31,25 & 37,5 & 43,7 & $50 \%$ & $>50 \%$ \\
\hline Necessidade de podas & $<37,5 \%$ & $37,5 \%$ & 46,9 & 56,3 & 65,7 & $75 \%$ & $>75 \%$ \\
\hline Desenvolvimento & $<22,5 \%$ & $22,5 \%$ & 28,2 & 34,0 & 39,0 & $45 \%$ & $>45 \%$ \\
\hline Sanidade & $<37,5 \%$ & $37,5 \%$ & 46,9 & 56,3 & 65,7 & $75 \%$ & $>75 \%$ \\
\hline Condição da raiz & $<47,5 \%$ & $47,5 \%$ & 59,4 & 71,3 & 83,2 & $95 \%$ & $>95 \%$ \\
\hline
\end{tabular}

\section{Obtenção da escala dos eixos do barômetro}

Para obter o maior valor possível da escala dos eixos do barômetro de sustentabilidade, os indicadores das equações 1 e 2 do método foram substituídos por 10, que representa a melhor condição de sustentabilidade.

Tendo todos os indicadores que compõem as equações alcançado a pontuação 10 , o valor máximo alcançado em cada eixo é igual a 100. Foi adotado o valor mínimo igual a 0 , conforme Braga et 
al. (2004a). Logo, obteve-se uma amplitude entre 0 e 100, sendo o intervalo entre eles dividido em quatro parte iguais, conforme figura 1.

\begin{tabular}{|c|c|c|c|c|c|}
\hline & \multicolumn{5}{|l|}{ Macroambientais } \\
\hline \multirow{5}{*}{ 递泀 } & & Muito baixo (0-25) & Baixo (26-50) & Bom (51-75) & Alto (76-100) \\
\hline & Alto (100-76) & & & & \\
\hline & Bom (75-51) & & & & \\
\hline & Baixo (50-26) & & & & \\
\hline & Muito baixo (25-0) & & & & \\
\hline
\end{tabular}

\begin{tabular}{|l|}
\hline Legenda \\
\hline Sustentável \\
\hline $\begin{array}{l}\text { Potencialmente } \\
\text { sustentável }\end{array}$ \\
\hline $\begin{array}{l}\text { Potencialmente } \\
\text { insustentável }\end{array}$ \\
\hline Insustentável \\
\hline
\end{tabular}

Figura 1. Barômetro de sustentabilidade aplicado ao método adaptado de Braga et al. (2004b). Figure 1. Barometer of sustainability applied to the suitable method of Braga et al. (2004b).

\section{Aplicação do método nas áreas testes}

Jardim Botânico

Para avaliação dessa área, segundo o método proposto, foi necessário obter dados primários para os indicadores que compõem as equações 4.2 e 4.3. Para isso, foi utilizada uma ortofoto na escala 1:10.000, do ano de 2002, que compreende a totalidade da área do parque junto à área reservada para o velódromo, conforme figura 2.

\section{Campus da Universidade Positivo}

Para avaliação dessa área, segundo o método proposto, foi necessário obter dados primários para os indicadores que compõem as equações 4.2 e 4.3. Para isso, foi utilizada uma ortofoto na escala 1:10.000, do ano de 2002, que compreende a totalidade da área do campus, conforme figura 3.

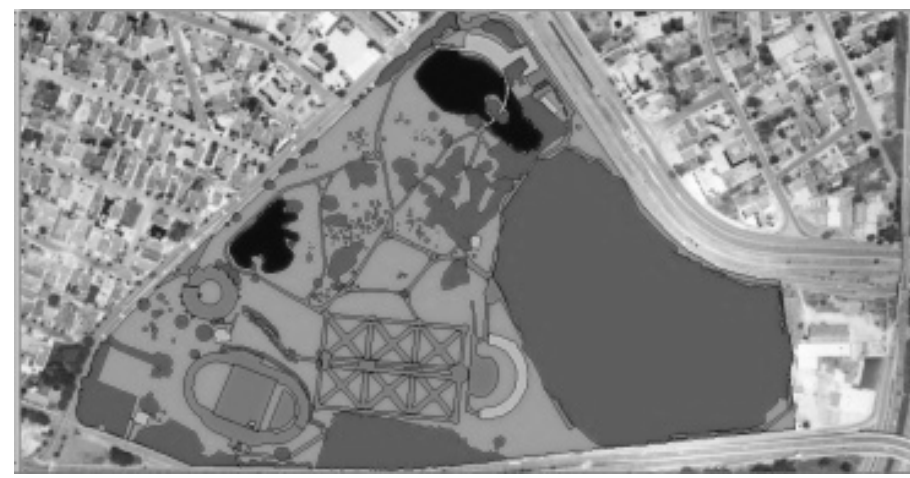

Figura 2. Imagem total do Jardim Botânico tratada pelo Arc View 3.2a.

Figure 2. Total image of the Botanic Garden treated by Arc View 3.2a.

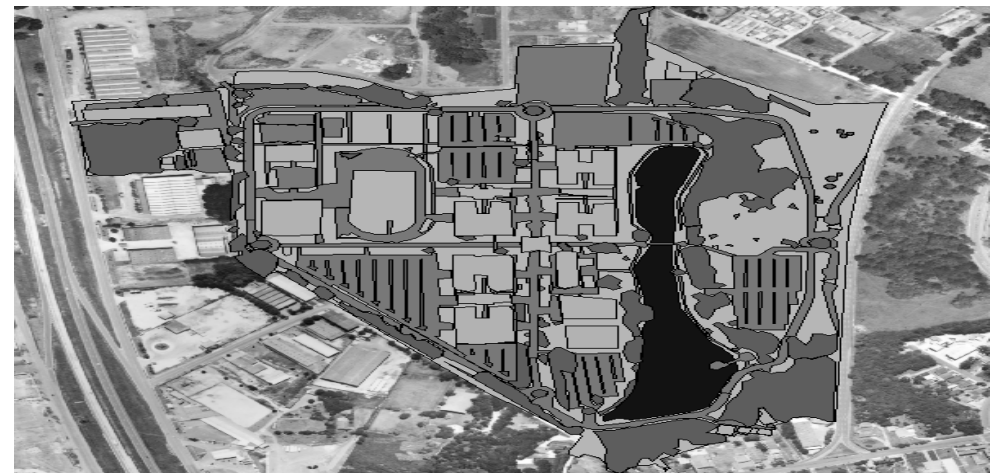

Figura 3. Imagem total da Universidade Positivo tratada pelo Arc View 3.2a.

Figure 3. Total image of the Positivo University treated by Arc View 3.2a. 
Depois de demarcados os elementos componentes, foram obtidas as áreas correspondentes, que estão descritas na tabela 5 .

Tabela 5. Dados coletados através do Arc View 3.2a.

Table 5. Collected datas through Arc View 3.2.

\begin{tabular}{lcc}
\hline & Jardim Botânico & Universidade Positivo \\
\hline Vegetação arbórea & $100.232 \mathrm{~m}^{2}$ & $90.476 \mathrm{~m}^{2}$ \\
Áreas impermeabilizadas & $29.747 \mathrm{~m}^{2}$ & $138.704 \mathrm{~m}^{2}$ \\
Lagos & $9.509 \mathrm{~m}^{2}$ & $29.203 \mathrm{~m}^{2}$ \\
Construções & $4.073 \mathrm{~m}^{2}$ & $41.602 \mathrm{~m}^{2}$ \\
Vegetações rasteiras & $87.435 \mathrm{~m}^{2}$ & $126.144 \mathrm{~m}^{2}$ \\
\hline Área total & $230.995 \mathrm{~m}^{2}$ & $426.129 \mathrm{~m}^{2}$ \\
\hline
\end{tabular}

Os outros indicadores que compõem o método foram obtidos através dos dados fornecidos por Nascimento (2005) e pela prefeitura Municipal de Curitiba. Os valores obtidos estão apresentados na tabela 6 .

Tabela 6. Informações coletadas por inventários e questionários.

Table 6. Collected informations by inventory and questionary.

\begin{tabular}{lcc}
\hline & Jardim Botânico & Universidade Positivo \\
\hline Usuários & $137^{* * *}$ & 10.000 \\
VC & $85 \%$ & $80 \%$ \\
Frequência da espécie mais abundante & $25,5 \% *$ & $14 \%$ \\
Densidade & $5.9 \mathrm{hab}^{*} \mathrm{ha}^{-1}$ & 235 hab.ha $^{-1}$ \\
Necessidade de poda & $95 \%$ & $99 \%$ \\
DAP médio & $90 \% *$ & $80 \%$ \\
Sanidade & $94 \%$ & $90 \%$ \\
Raiz & $98 \%$ & $100 \%$ \\
\hline *Dado obtido do levantamento fitossociológico de Nascimento (2005). **Dado obtido na Prefeitura Municipal de Curitiba (2007).
\end{tabular}

\section{Cálculos para o diagnóstico do Jardim Botânico}

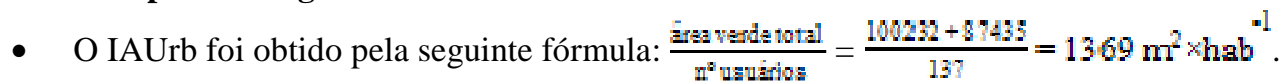

- O índice Di foi fornecido por Nascimento (2005), conforme tabela 4.11. O Di corresponde a 25,5\%.

- O PAV foi obtido pela seguinte fórmula: $\frac{\mathrm{ELA}}{\mathrm{ELA} A \mathrm{ELH}}=\frac{100232}{10023+8743 \%}=53 \%$.

- O índice Densidade Populacional foi obtido pela seguinte fórmula: $\frac{\mathrm{Hab}}{\text { areatotal }}=\frac{137}{23,0995}=5.9 \mathrm{hab}$. ha $\mathrm{A}^{-1}$.

- O índice IFU foi obtido pela seguinte fórmula: $\frac{\mathrm{ILA}}{\mathrm{ILA}+\mathrm{IC}+\mathrm{II}}=\frac{100232}{100232+29747+4073}=75 \%$.

- O índice Valor de Contingência foi obtido através de questionário aplicado na área. VC corresponde a $85 \%$.

- Necessidade de poda ( $\mathrm{Np}$ ) foi obtido através de inventário, atingindo um valor de $95 \%$ dos indivíduos analisados que não precisavam de podas.

- Desenvolvimento (De) foi obtido através de inventário, atingindo um valor de $90 \%$ da população arbóreas com DAP acima de $15 \mathrm{~cm}$.

- Sanidade foi obtido através de inventário, atingindo um valor de $94 \%$ da população arbórea sem apresentar problemas de sanidades significativos.

- Condição da Raiz (Cr) foi obtido através de inventário, atingindo um valor de 96,5\% das raízes com padrões normais.

Os dados da tabela 7 foram submetidos a padronização, conforme a escala apresentada na tabela 4. Seus valores foram substituídos pelos valores $0,1,3,5,7,9$ e 10, de acordo com a proximidade dos valores extremos. 
Tabela 7. Resultado dos indicadores do Jardim Botânico.

Table 7. Result from the Botanic Gardem indicators.

\begin{tabular}{ll}
\hline Indicadores & Valores obtidos através da escala de padronização \\
\hline IAUrb & $1369 \mathrm{~m}^{2}$ de áreas verdes por habitante. \\
Diversidade & $25,5 \%$ para a espécie mais abundante. \\
PAV & $53,4 \%$ de áreas arborizadas no total de áreas verdes. \\
DP & 5.9 hab.ha $^{-1}$. \\
IFU & $75 \%$ de espaço arborizado em relação à área construída. \\
VC & $85 \%$ das pessoas entrevistadas se disponibilizariam a pagar pelo serviço \\
Necessidade de podas & prestado pela vegetação naquele local. \\
Desenvolvimento & Em $95 \%$ das árvores verificadas não havia necessidade. \\
Sanidade & $90 \%$ das árvores apresentaram DAP acima de 15 cm. \\
Condição da raiz & $94 \%$ das árvores verificadas não apresentaram problemas de sanidade \\
\hline
\end{tabular}

\section{Cálculos para o diagnóstico da Universidade Positivo}

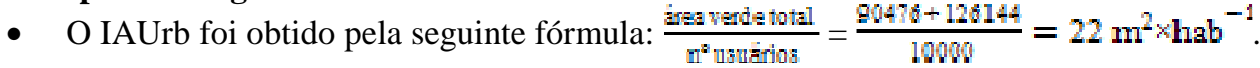

- O índice (Di) foi obtido através de inventário, atingindo um valor de $14 \%$.

- O PAV foi obtido pela seguinte fórmula: $\frac{\mathrm{ELA}}{\mathrm{ZIA}+\mathrm{ZLH}}=\frac{90476}{90476+126144}=42 \%$.

- O índice Densidade Populacional foi obtido pela seguinte fórmula: $\frac{\mathrm{Hab}}{\text { arsatal }}=\frac{10000}{42.6 \mathrm{ag}}=235 \mathrm{hab} \times \mathrm{ha}^{-1}$.

- O índice IFU foi obtido pela seguinte fórmula: $\frac{E L A}{E L A+E C+E I}=\frac{90476}{90478+41002+138704}=34 \%$.

- O índice Valor de Contingência foi obtido através de questionário aplicado na área. VC corresponde a $80 \%$.

- Necessidade de poda (Np) foi obtida através de inventário, atingindo um valor de $99 \%$ dos indivíduos analisados que não precisavam de podas.

- Desenvolvimento (De) foi obtido através de inventário, atingindo um valor de $80 \%$ da população arbóreas com DAP acima de $15 \mathrm{~cm}$.

- Sanidade foi obtido através de inventário, atingindo um valor de $90 \%$ da população arbórea sem apresentar problemas de sanidades significativos.

- Condição da raiz $(\mathrm{Cr})$ foi obtido através de inventário, atingindo um valor de $100 \%$ das raízes com padrões normais.

Tabela 8. Resultados dos indicadores da Universidade Positivo.

Table 8. Result from the Positivo University indicators.

\begin{tabular}{ll}
\hline Indicadores & Valores obtidos através da escala de padronização \\
\hline IAUrb & $22 \mathrm{~m}^{2}$ de áreas verdes por habitante. \\
Diversidade & $14 \%$ para a espécie mais abundante. \\
PAV & $42 \%$ de áreas arborizadas no total de áreas verdes. \\
DP & 235 hab.ha $^{-1}$. \\
IFU & $34 \%$ de espaço arborizado em relação à área construída. \\
VC & $80 \%$ das pessoas entrevistadas se disponibilizariam a pagar pelo serviço prestado \\
Necessidade de podas & pela vegetação naquele local. \\
Desenvolvimento & Em $99 \%$ das árvores verificadas não havia necessidade. \\
Sanidade & $80 \%$ das árvores analisadas apresentaram DAP acima de 15 cm. \\
Condição da raiz & $90 \%$ das árvores verificadas não apresentaram problemas de sanidade significativos \\
\hline
\end{tabular}

Os resultados das tabelas 7 e 8 foram submetidos a padronização, conforme a escala apresentada na tabela 4. $\mathrm{O}$ resultado desse processo é demonstrado na tabela 9. 
Tabela 9. Aplicação da escala de padronização segundo os resultados dos indicadores.

Table 9. Aplication of the scale of padronization.

\begin{tabular}{lcc}
\hline \multirow{2}{*}{ Indicadores } & \multicolumn{2}{c}{ Valores obtidos através da escala de padronização } \\
\cline { 2 - 3 } & Jardim Botânico & Universidade Positivo \\
\hline IAUrb & 10 & 10 \\
Diversidade & 0 & 10 \\
IFU & 10 & 10 \\
DP & 10 & 10 \\
PAV & 10 & 10 \\
VC & 10 & 10 \\
Necessidade de podas & 10 & 10 \\
Desenvolvimento & 10 & 10 \\
Sanidade & 10 & 10 \\
Condição da raiz & 10 & 10 \\
\hline
\end{tabular}

\section{Aplicação das equações}

Os valores já padronizados (tabela 10) são aplicados nas equações 1 e 2, para obtenção dos valores macro e microambientais, que correspondem aos dois eixos do barômetro de sustentabilidade.

Jardim Botânico

$\mathrm{VMaA}=3 \times 10+2 \times 10+2 \times 0+2 \times 10+10=80$

$\mathrm{VMiA}=3 \times 10+3 \times 10+2 \times 10+10+10=100$

Universidade Positivo

$\mathrm{VMaA}=3 \times 10+2 \times 10+2 \times 10 \mathrm{x}+2 \times 10+10=100$

$\mathrm{VMiA}=3 \times 10+3 \times 10+2 \times 10+10+10=100$

\begin{tabular}{|c|c|c|c|c|c|c|}
\hline & \multicolumn{6}{|l|}{ Macroambientais } \\
\hline \multirow{5}{*}{ 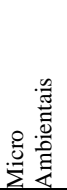 } & & Muito baixo (0-25) & Baixo (26-50) & Bom (51-75) & & -100) \\
\hline & Alto (100-76) & & & & $\mathrm{JB}$ & UP \\
\hline & \multicolumn{6}{|l|}{ Bom (75-51) } \\
\hline & Baixo (50-26) & & & & & \\
\hline & \multicolumn{6}{|l|}{ Muito baixo (25-0) } \\
\hline
\end{tabular}

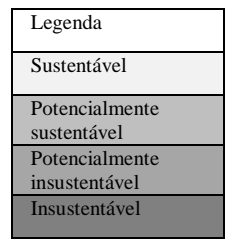

Figura: 4. Barômetro de sustentabilidade diagnóstico das áreas.

Figure 4 . Barometer of sustainability diagnose areas.

\section{Resultados da aplicação do barômetro no Jardim Botânico}

$\mathrm{Na}$ avaliação da área do Jardim Botânico por esse método, constatou-se uma condição sustentável. O resultado refletiu a pequena dimensão de áreas construídas, a impermeabilização que basicamente consiste em ciclovias e passarelas, as extensas áreas de gramados, a área de remanescente, composta pelo bosque junto a faixas arborizadas, que compreendem aproximadamente $43 \%$ do total da área analisada.

Os aspectos microambientais avaliados também apresentaram condições favoráveis, como um desenvolvimento de $90 \%$ das plantas com DAP acima de $10 \mathrm{~cm}$, sendo os $10 \%$ que obtiveram valor inferior, provavelmente resultado de plantios recentes ou de espécies de crescimento mais lentos. Os problemas com as raízes foram constatados em três indivíduos localizados próximos às cercas limites, beirando a ciclovia, possível reflexo da arborização de rua sem preocupação com desenvolvimento das raízes. As demais, no entanto, estavam em perfeitas condições, segundo os critérios analisados. Quanto ao índice Valor de Contingência, apenas duas pessoas não se mostraram dispostas a pagar a quantia de $\mathrm{R} \$ 1,00$ pelos serviços prestados pela floresta urbana, contudo, todas responderam sim a uma das três perguntas feitas, o que demonstra certo grau de indecisão.

Todavia, cabe ressaltar que se trata de uma ferramenta que avalia uma série de elementos das 
florestas urbanas, permitindo, dessa maneira, que condições sustentáveis possam ser atingidas mesmo em áreas fortemente antropizadas.

Por essa razão, é possível imaginar que, quando aplicado o método em fragmentos de áreas urbanas com características paisagísticas de parques ou praças, os posicionamentos dessas áreas dentro do barômetro de sustentabilidade tenderão ao sustentável.

\section{Resultados da aplicação do barômetro no Campus da Universidade Positivo}

No Campus da Universidade Positivo, o diagnóstico constatou uma condição sustentável, de forma semelhante à obtida no Jardim Botânico. O resultado reflete a extensa área permeável composta por gramados e a área composta pelo bosque junto a faixas arborizadas, que compreende aproximadamente $22 \%$ do total da área analisada, uma porção ainda expressiva.

Os aspectos microambientais avaliados também apresentaram condições favoráveis, como um desenvolvimento apresentando $80 \%$ das plantas com DAP acima de $10 \mathrm{~cm}$, sendo os $20 \%$ que obtiveram valor inferior, provavelmente resultado de plantios recentes. Não foram constatados problemas com as raízes, devido à existência de preocupação no planejamento dessas áreas com o desenvolvimento e a influência das raízes nas áreas construídas e na velocidade de crescimento da arborização do local.

Quanto ao índice Valor de Contingência, apenas três pessoas não se mostraram dispostas a pagar a quantia de $\mathrm{R} \$ 1,00$ pelos serviços prestados pela floresta urbana, contudo, um respondeu sim para uma das três perguntas feitas e as outras duas responderam não para as três perguntas, o que demonstra certo grau de indecisão em uma das três pessoas. Entretanto, as respostas desfavoráveis a esse pagamento podem ser consequência do entendimento de que esse valor já estivesse embutido nas mensalidades, por tratar-se de alunos nessas duas situações.

Cabe ressaltar que o Campus da Universidade Positivo foi planejado com o intuito de buscar uma melhor interação entre os espaços edificados e os elementos que compõem a floresta urbana.

\section{CONCLUSÕES}

- Vários são os indicadores e parâmetros apresentados na literatura como ferramentas para analisar a qualidade das florestas urbanas. Todavia, as suas utilizações se fundamentam na escolha dos parâmetros que são observados e na interpretação dos dados obtidos pela sua utilização, apoiando-se, na maioria das vezes, na interpretação subjetiva de profissionais, quando utilizados para responder questionamentos sobre a sustentabilidade dessas áreas. Cabe ressaltar que as interpretações da condição de sustentabilidade são complexas e necessitam da leitura e da compreensão de um grande número de variáveis.

- A etapa da pesquisa e seleção de indicadores apresentou algumas dificuldades, como a repetição dos critérios de avaliação por vários indicadores, a variação de conceitos utilizados pelos mesmos índices, a variação de conceitos no emprego dos temas indicadores e nos termos que se referem à arborização urbana.

- Contudo, o ponto crítico foi no estabelecimento dos valores máximos e mínimos, pela carência de parâmetros de referência, sendo estes, em muitos casos, estabelecidos no diagnóstico de áreas específicas, dificultando a transposição para uma condição mais global. Todavia, abre-se um precedente para novas pesquisas, sendo tal necessidade evidenciada e mencionada em muitos trabalhos.

- Cabe salientar a importância de uma metodologia que utilize valores de referência teóricos ou máximos e mínimos, permitindo uma maior amplitude do poder de comparação, o que permite uma maior clareza na leitura por parte de gestores e pela própria sociedade, não tão habilitada no que tange à complexidade das ciências que demarcam esse meio, conhecido como florestas urbanas.

- O método proposto mostrou-se capaz de interagir e ponderar tais indicadores e auxiliar na construção de um diagnóstico mais claro e pontual. Porém algumas condições delimitam e podem comprometer tal diagnóstico, como, por exemplo, a quantidade de tabelas avaliadas por pesquisadores, professores e especialistas, a determinação dos valores máximos e mínimos, influenciada por suas divergências e inexistência de dados, na maioria dos casos fazendo-se necessário a sua estipulação com base no entendimento de poucas referências.

- A proposição de qualquer método tem que considerar a flexibilização na modificação e avaliação temporal dos indicadores que o compõem, considerando sempre a dinâmica da ciência e dos meios urbanos e da própria floresta urbana. 


\section{REFERÊNCIAS}

BADIRU, A. I.; PIRES, M. A.; RODRIGUEZ, A. M. Método para classificação tipológica da floresta urbana visando o planejamento e a gestão de cidades. In: SIMPÓSIO BRASILEIRO DE SENSORIAMENTO REMOTO, 12., 2005, Goiânia. Anais... Goiânia, 2005. p. 1427 - 1433.

BERTÉ, G. A.; KARNAUKHOVA, E.; LOCH, C. Cenários de implantação do sistema de áreas verdes com base nos instrumentos de gestão ambiental propostos no âmbito de um novo plano diretor e Código de Meio Ambiente. In: CONGRESSO BRASILEIRO DE CADASTRO TÉCNICO MULTIFINALITÁRIO, 2006, Florianópolis. Anais do... Florianópolis: UFSC, 2006.

BRAGA, T. M.; FREITAS, A.; DUARTE, G.; SOUSA, J. Indicadores de sustentabilidade municipal: o desafio de mensurar. Belo Horizonte: UFMG/Cedeplar, 2004a. 22 p.

BRAGA, T. M.; BRITO, F.; FREITAS, A.; MARQUES, D. Sustentabilidade e condições de vida em áreas urbanas: medidas e determinantes nas Regiões Metropolitanas de São Paulo e Belo Horizonte. In: ENCONTRO NACIONAL DE ESTUDOS POPULACIONAIS, 14., 2004, Caxambú, MG. Anais do... Caxambú: ABEP, 2004b.

CARVALHO, L. M. Áreas verdes da cidade de Lavras/MG: caracterização, uso e necessidade. 115 p. Dissertação (Mestrado em Fitotecnia) - Universidade Federal de Lavras, Lavras, 2001.

DETZEL, V. A. Arborização urbana: importância e avaliação econômica. In: CONGRESSO BRASILEIRO SOBRE ARBORIZAÇÃO URBANA, 1., 1992, Vitória, ES. Anais do... Vitória, 1992. p. 39 - 52.

FERREIRA, I. L.; GONTIJO, B. M. Um histórico verde: a retração da vegetação remanescente no município de Belo Horizonte. In: SIMPÓSIO BRASILEIRO DE GEOGRAFIA FÍSICA APLICADA, 11., 2005, São Paulo. Anais do... São Paulo: USP, 2005.

FIALHO, I. C.; CASSIA, H. R.; RIBEIRO, S.; TAVARES, A. Indicadores de áreas verdes e cobertura vegetal para as praças do município de Vinhedo, SP. Revista Árvore, Viçosa, v. 30, n. 2, p. 277 - 282, 2006.

GREY, G. W.; DENEKE, F. J. Urban forestry. New York: J. Willey, 1986. 279 p.

HILDEBRAND, E. Avaliação econômica dos benefícios gerados pelos parques urbanos: estudo de caso em Curitiba, PR. 137 p. Tese (Doutorado em Ciências Florestais) - Setor de Ciências Agrárias, Universidade Federal do Paraná, Curitiba, 2001.

JESUS, S. J.; BRAGA, R. Análise espacial das áreas verdes urbanas da Estância de Águas de São Pedro, SP. Caminhos de Geografia. v. 18, p. 207 - 224, 2005.

LIMA, N. E. M.; RESENDE, W. X.; SENA, M. G. D. Análise das áreas verdes das praças do bairro centro e principais avenidas da cidade de Aracaju, SE. Revista da Sociedade Brasileira de Arborização Urbana, v. 2, n. 1, p. 17 - 33, 2007.

LIMA, A. M. L. P.; COUTO, H. T. Z; ROXO, J. L. C. Análise de espécies mais frequentes da arborização viária, na zona urbana central do município de Piracicaba/SP. In: CONGRESSO BRASILEIRO DE ARBORIZAÇÃO URBANA, 2., 1994, São Luís. Anais... São Luís, 1994. p. 555 573.

MELO, R. R.; LIRA FILHO, J. A.; RODOLFO JÚNIOR, F. Diagnóstico qualitativo e quantitativo da arborização urbana no bairro Bivar Olinto, Patos, Paraíba. Revista da Sociedade Brasileira de Arborização Urbana, v. 2, n. 1, p. 64 - 80, 2007.

MILANO, M. S. Avaliação quali-quantitativa e manejo da arborização urbana: exemplo de Maringá. 120 p. Tese (Doutorado em Engenharia Florestal) - Universidade Federal do Paraná, Curitiba, 1988. 
Planejamento da arborização urbana: relação entre áreas verdes e ruas arborizadas. In: ENCONTRO NACIONAL SOBRE ARBORIZAÇÃ̃O URBANA, 3., 1990, Curitiba. Anais do... Curitiba: FUPEF, 1990. p. $60-71$.

MILANO, M. S.; DALCIN, E. Arborização de vias públicas. Rio de Janeiro: Light, 2000. 206 p.

MOREIRA, T. C. L.; SILVA, F. D. F.; POLIZEL, J. L. Extração de cobertura arbórea intraurbana de imagens de alta resolução. In: SIMPÓSIO BRASILEIRO DE SENSORIAMENTO REMOTO, 13., 2007, Florianópolis. Anais... Florianópolis: INPE, 2007. p. 5403 - 5409.

NASCIMENTO, D. S. Levantamento da vegetação fanerogâmica do Jardim Botânico Municipal de Curitiba. Monografia (Graduação em Ciências Biológicas) - Centro Universitário Positivo, Curitiba, 2005.

OLIVEIRA, C. H. Planejamento ambiental na cidade de São Carlos (SP) com ênfase nas áreas públicas e áreas verdes: diagnósticos e propostas. $181 \mathrm{f}$. Dissertação (Mestrado em Ecologia e Recursos Naturais) - Universidade Federal de São Carlos, São Carlos, 1996.

PAIVA, H. N.; GONÇALVEZ, W. Florestas urbanas: planejamento para melhoria da qualidade de vida. Aprenda Fácil, v. 2, Viçosa, 2002.

PREFEITURA MUNICIPAL DE CURITIBA. Plano municipal de controle ambiental e desenvolvimento sustentável: diagnóstico III versão. Curitiba: PMC, 2007. 359 p.

SILVA, A. G. Avaliação da arborização no perímetro urbano de Cajuri - MG, pelo método da tabela sintético. 150 p. Dissertação (Mestrado em Ciência Florestal) - Universidade Federal de Viçosa, Viçosa, 2000.

SILVA, A. G.; GONÇALVES, W.; LEITE, H. G.; SANTOS, E. Comparação de três métodos de obtenção de dados para avaliação quali-quantitativa da arborização viária em Belo Horizonte, MG. Revista da Sociedade Brasileira de Arborização Urbana, v. 1, n. 1, p. 31 - 44, 2006.

SILVA, F. A aplicação de videografia aérea multiespectral na avaliação de floresta urbana. $88 \mathrm{p}$. Tese (Doutorado em Agronomia - Produção Vegetal) - Universidade Estadual Paulista, Faculdade de Ciências Agrárias e Veterinárias, Jaboticabal, 2004.

SEITZ, R. A. Manual da poda de espécies arbóreas florestais. Curitiba: FUPEF. 1995.

TAKAHASHI, L. Y. Arborização urbana: inventário. In: CONGRESSO BRASILEIRO SOBRE ARBORIZAÇÃO URBANA, 2., 1994, São Luiz. Anais do... São Luiz: SBAU, 1994. p. 193 - 199.

TOMASONI, M. A. Contribuição ao estudo de indicadores ambientais. Geonordeste, ano 15, n. 2, 2006. p. $90-118$. 
FLORESTA, Curitiba, PR, v. 41, n. 3, p. 501-518, jul./set. 2011.

Mazzarotto, A. de S.; Cubas, S.; Maranho, L. T. 